\title{
Three adjoints to the fundamental theorem of physics
}

\section{Abstract}

Astrotheology provides for the underlying mathematical truths of our universe. We see that the derivative puzzle provides an insight into how the golden mean, the derivative equals the function; and velocity equals acceleration. Its' important because all of physics rest of these mathematical principles.

Keywords: astrotheolgy, fundamental theorems of physics, at math, derivatives
Volume I Issue 4 - 2018

\section{Paul TE Cusack}

Park Ave, Saint John, Canada

Correspondence: Paul TE Cusack, BScE, Dule 23 Park Ave, Saint John, NB E2J IR2, Canada, Tel 5066526350 Email st-michael@hotmail.com

Received: July 30, 2018 | Published: August 16,2018

\section{Introduction}

The Fundamental Theorems of Physics is described by one writer as: The real and physical part of the universe can be described by the mean of mathematics. In this paper we provide three clarifying additions to this theorem that result for considering Astrotheology Mathematic and calculus (Figure 1).

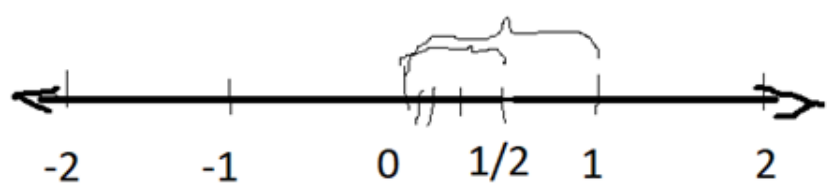

Figure 1 The number line showing the $1 / 2$ of a distance dilemma.

Let $y=y^{\prime}$ or $f^{\prime}(x)=f(x)$

The solution to this Differential Equation is:

$y=e^{x}$

If we take the number line, and move half way from 1 to 0 , we get $1 / 2$. If we move half way again from $1 / 2$ to 0 , we get $1 / 4$ etc. We never actually reach 0 because we can always take $1 / 2$ of a number.

The solution to this dilemma is that as we move from 1 to $1 / 2$, we also move from 0 to $1 / 2$. So when $1 / 2=1 / 2$ we get to the half way point. Problem solved (Figure 2).

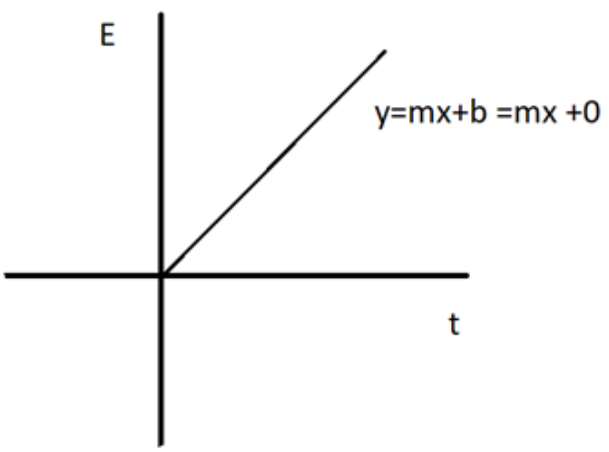

Figure 2 The slope of a linear function.

$y=y^{\prime}$

$1 / 2=1 / 2$ they meet.

$$
\begin{aligned}
& x-x=0=h \\
& y=y^{\prime}=e^{1 / 2} \\
& \text { Ln } y=\operatorname{Ln} y^{\prime}=1 / 2 \text { Lne } \\
& \text { Ln } y^{\prime}=1 / 2 \\
& y=e^{1 / 2} \\
& x=1 / 2 \\
& f(t+h)=m t=t \\
& f(x)=y=m x \\
& {[(m+h) x-m x] / h=1} \\
& m=1 \\
& {[(1+h) x-x] / h=1} \\
& x=t=1 \\
& {[h-1] / h=1} \\
& h=0 \\
& f^{\prime}(x)=\lim { }_{h \mapsto 0}[f(x+h)-f(x)] / h
\end{aligned}
$$

Let $x=1 / 2$

$=[f(1 / 2+h)-f(-1 / 2)] / h$

$[f(1 / 2)-f(-1 / 2)+h] / h$

$=1=E=t$

$y^{\prime}=1$

$y^{\prime}=x^{0}$

slope $m=1$

$y=y^{\prime}$ Fundamental Theorem of Physcis Adjoint 1.

$f^{\prime}(x)=\lim _{h \mapsto 0}[f(-1 / 2)+f(h)-f(1 / 2)] / h$ 


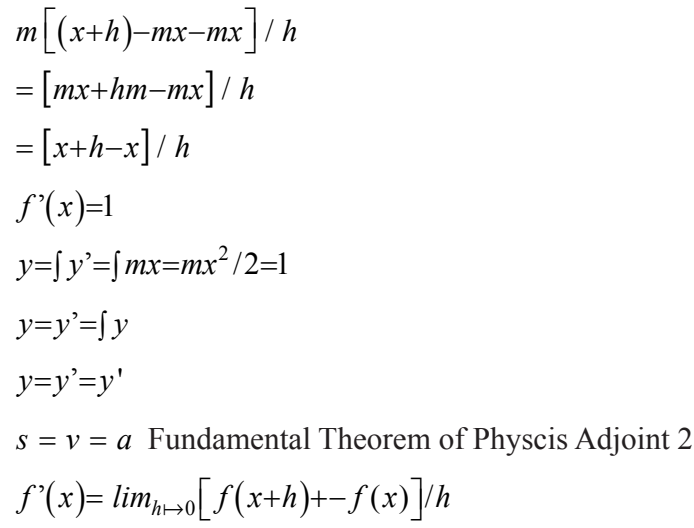

But $h \mapsto 0$ Therefore we have the Associative Law for functions.

$f^{\prime}(x)=m+m h-(m(f(-x))$

$f^{\prime}(x)=m x+m h+m x$

$f(x)=2 m+m h$

$f(x)=2 x-1$

This is the derivative of the golden mean function.

$\int f^{\prime}(x)=y=2 x^{2}-x$
But $y=1$

$2 x^{2}-x-1=0$

$x^{2}-x-1=0$ The Fundamental Theorem of Physics Adjoint 3

\section{Conclusion}

We see that Astrotheology Mathematics (AT Math) provides the mathematician to develop the fundamental theorem to explain the fundamental principles underlying all of physics or at least the known universe.

\section{Acknowledgements}

None.

\section{Conflict of interest}

The author declares that there is no conflict of interest.

\section{References}

1. Cusack P. Astrotheology, Cusack's Universe. J of Phys Math. 2016;7(2):8.

2. Stewart Ian. In Pursuit of the Unknown. Basic Books. 2012; p. 352.

3. LeCornac H. Fundamental Theorems of Physics. 2017. 